\title{
Short spatial resolutions retrieval from a long pulse BOTDA trace
}

Sheng Wang, Zhisheng Yang, Simon Zaslawski, Luc Thévenaz

Sheng Wang, Zhisheng Yang, Simon Zaslawski, Luc Thévenaz, "Short spatial resolutions retrieval from a long pulse BOTDA trace," Proc. SPIE 11199, Seventh European Workshop on Optical Fibre Sensors, 111992B (28 August 2019); doi: $10.1117 / 12.2540927$

Event: Seventh European Workshop on Optical Fibre Sensors, 2019, Limassol, Cyprus 


\title{
Short spatial resolutions retrieval from a long pulse BOTDA trace
}

\author{
Sheng Wang ${ }^{\mathrm{a}, \mathrm{b}}$, Zhisheng Yang ${ }^{* a}$, Simon Zaslawski and Luc Thévenaz ${ }^{\mathrm{a}}$ \\ ${ }^{a}$ EPFL Swiss Federal Institute of Technology, Group for Fibre Optics, SCI STI LT Station 11, 1015 \\ Lausanne, Switzerland; 'Beijing University of Posts \& Telecommunications, 100876 Beijing, China. \\ *Email: zhisheng.yang@epfl.ch
}

\begin{abstract}
A novel technique is proposed to obtain a flexible and variable spatial resolution from a conventional Brillouin optical time-domain analyzers using a fast post-processing algorithm. The approach is very attractive since a fine spatial resolution can be obtained from a coarsely resolved measurement obtained using a pulse longer than the acoustic settling time, leading to a better overall sensing performance, in particular for sub-metric spatial resolutions, with no compromises on sensing range and measurement time.
\end{abstract}

Keywords: Optical Fiber Sensor, Stimulated Brillouin Scattering, distributed fiber sensing.

\section{INTRODUCTION}

Brillouin optical time-domain analysis (BOTDA) offers the possibility of distributed sensing over long distances and with high precision ${ }^{1}$. The Brillouin frequency shift (BFS) at any position along the fiber, which depends linearly on the local temperature/strain change, is estimated by post-processing the local Brillouin gain spectrum (BGS) reconstructed by scanning the pump-probe frequency difference. The BGS parameters, such as the peak gain and the full-width at half maximum (FWHM), determine the BFS accuracy. Since the BGS profile results from the convolution between the pump pulse spectrum and the fiber natural $\mathrm{BGS}^{2}$, the use of a shorter pump pulse leads to a broader FWHM and lower peak gain, drastically impairing super-linearly the sensor performance. For this reason, a limit for a good BFS accuracy is set for conventional BOTDAs as the situation when pump pulse and natural Brillouin gain spectra show a comparable FWHM, corresponding to a pump duration of $12 \mathrm{~ns}$, or about $1 \mathrm{~m}$ spatial resolution (SR). In order to overcome this trade-off between SR and BFS accuracy, a large quantity of time-domain ${ }^{3-5}$ and correlation-based techniques ${ }^{6}$ have been developed, unavoidably compromising on other specifications such as measurement time $\mathrm{e}^{3,4}$ or sensing range ${ }^{5,6}$.

In this paper, we propose a simple alternative to retrieve a target SR by simply post-processing measurements obtained using a conventional BOTDA with a fixed long pump pulse. The proposed technique is immune to the negative impact of the acoustic inertial response, overcoming the traditional trade-off between SR and BFS accuracy in BOTDA, while keeping the same measurement time and sensing range. The technique is only compromised by the predictable BFS errors appearing prior to the edge of hotspots, which may be reduced by using more sophisticated algorithm. $2 \mathrm{~m}, 1 \mathrm{~m}$ and $0.2 \mathrm{~m}$ SRs are experimentally demonstrated using long pump pulses, outperforming standard BOTDAs.

\section{PRINCIPLE}

In a standard BOTDA, the sensing fiber is interrogated by a CW probe and an isolated pump pulse $p(t)$, where the pulse duration $T_{p}$ determines the SR. In this case the obtained time-domain trace $r_{p}(t)$ is represented by the linear convolution between $p(t)$ and the fiber impulse response $h(t)$, merged with an additive noise $e(t)$ :

$$
\mathrm{r}_{\mathrm{p}}(t)=p(t) \otimes h(t)+e(t) \stackrel{F F T}{\longrightarrow} R_{p}(f)=P(f) \cdot H(f)+E(f)
$$

where $\otimes$ stands for a linear convolution. Eq. (1) also shows the dual representation in the frequency domain, where $R_{p}(f)$, $H(f)$ and $E(f)$ are the spectra of $p(t), h(t)$ and $e(t)$, respectively. Here we treat the long pump pulse as formed by a concatenation of $N$ short pulse segments with identical duration $T_{S}\left(=T_{p} / N\right)$ that corresponds to the targeted SR. This way $p(t)$ can be further represented by the convolution between one elementary short pulse $s(t)$ and a series of delta functions $d(t)$ that are equally spaced by $T_{s}$ for a total number of $N$, as illustrated in Fig. 1.

Seventh European Workshop on Optical Fibre Sensors, edited by Kyriacos Kalli, Gilberto Brambilla, Sinead O'Keeffe Proc. of SPIE Vol. 11199, 111992B · @ 2019 SPIE · CCC code: 0277-786X/19/\$21 · doi: 10.1117/12.2540927 

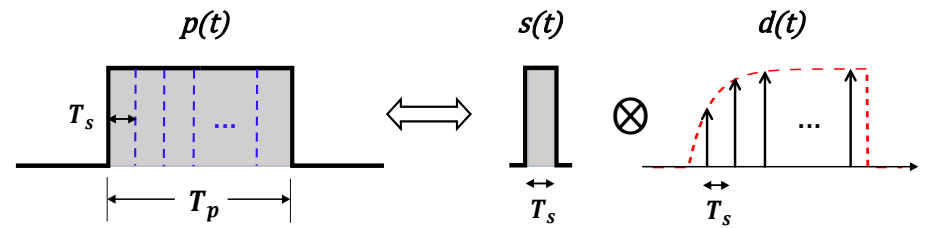

Figure 1. Illustration of the pulse decomposition concept and the equivalent convolution process.

Note that due to the inertial feature of the acoustic wave in BOTDA, each delta function in $d(t)$ contributes differently, following an envelope (red dashed curve in Fig. 1) that can be analytically expressed as ${ }^{5}$ :

$$
\text { envelope }(\mathrm{t})=\left[1-\exp \left(-\frac{t}{2 \tau_{A}}\right)\right] \cdot\left[u(t)-u\left(t-T_{p}\right)\right]
$$

where $\tau_{\mathrm{A}}$ is the acoustic settling time ( $6 \mathrm{~ns}$ in standard silica fibers) and $\mathrm{u}(\cdot)$ stands for the Heaviside unit step function. This way Eq. (1) can be rewritten as:

$$
\mathrm{r}_{\mathrm{p}}(t)=[s(t) \otimes d(t)] \otimes h(t)+e(t) \stackrel{F F T}{\longrightarrow} R_{p}(f)=S(f) \cdot D(f) \cdot H(f)+E(f)
$$

where $S(f)$ and $D(f)$ represent the spectra of $s(t)$ and $d(t)$, respectively. Based on Eq. (3), the short-pulse response $r_{s}(t)$ can be retrieved by performing a deconvolution in the frequency domain associated with an IFFT process:

$$
r_{s}(t)=\operatorname{IFFT}\left[\frac{R_{p}(f)}{D(f)}\right]=\operatorname{IFFT}\left[S(f) \cdot H(f)+\frac{E(f)}{D(f)}\right]=s(t) \otimes h(t)+\operatorname{IFFT}\left[\frac{E(f)}{D(f)}\right]
$$

in total similarity to the decoding process reported in ref. 7. Note that the negative impact of the acoustic inertial behavior is cancelled out during the post-processing, so that the retrieved $r_{s}(t)$ actually represents the Brillouin response under the steady-state condition regardless of the targeted SR. This means that the post-processed BGS will exhibit a Lorentzian profile showing the Brillouin natural linewidth $(\sim 30 \mathrm{MHz})$ and the peak Brillouin gain will depend linearly on the target SR, as confirmed by the simulated results shown in Fig. 2(a), for 2-m and 1-m SRs (blue and red solid curves) retrieved from 6-m SR data. Compared to BGS obtained by conventional single-pulse BOTDA with duration set for the same SR (black and green dashed curves in Fig. 2(a)), post-processed BGS present much larger amplitude and narrower BGS FWHM, leading to a better BFS accuracy ${ }^{8}$. It is worth mentioning that the mean value of the term $1 / D(f)$ in Eq. (4), which represents the noise magnification factor ${ }^{7}$, is calculated to be $\sim 1.5$ for any SR, penalizing the response accordingly. Taking all these aspects into account, the SNR and the BFS accuracy improvements by the proposed technique over the conventional single-pulse BOTDA, as a function of SR, are shown in Fig. 2(b). Larger improvements can be obtained for sharper SRs, corresponding to cases more affected by the acoustic transient effects.
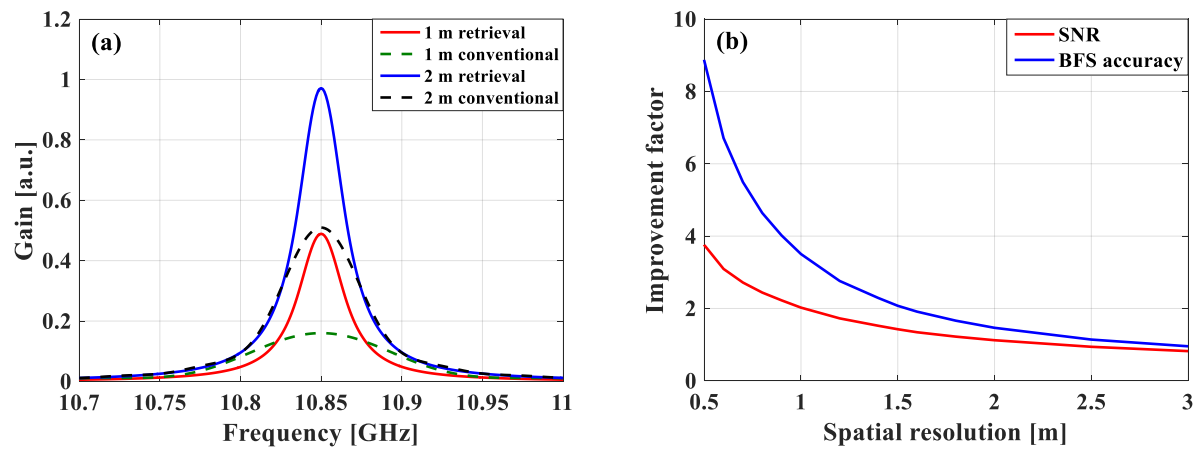

Figure 2. (a) Simulated BGS using proposed technique and conventional BOTDA; (b) BFS accuracy (blue) and SNR improvement (red) as a function of spatial resolution by the proposed technique with respect to the classical approach.

It should be noted that BFS determination errors may appear at fiber sections prior to hotspots, as will be shown in the experimental part of this paper. This is due to the fact that the envelope function (Eq. (4)) used in this method only describes precisely the situation at the Brillouin resonance frequency, while the functional dependence for detuned frequencies are actually slightly different. These systematic errors turn out to be max. $3 \mathrm{MHz}$, which may not be of 
crucial importance for some applications. Besides, since the error locations can be identified from the locations of hotspots, it is possible to process and reduce those BFS errors using more sophisticated algorithms.

\section{EXPERIMENTAL DEMONSTRATION AND DISCUSSION}

Experiments using a standard BOTDA are carried out to verify the feasibility of the proposed technique. Results are mainly investigated around a $2-\mathrm{m}$-long hotspot at $40{ }^{\circ} \mathrm{C}$, placed at the end of a $47.52 \mathrm{~km}$ long sensing fiber at room temperature $\left(27^{\circ} \mathrm{C}\right)$. First, a classical measurement is performed using a 60-ns long pump pulse, corresponding to an original SR of $6 \mathrm{~m}$. The 2D-mapping of the Brillouin gain distribution, as a function of fiber position and scanning frequency, is shown in Fig. 3(a), where the 2-m hotspot cannot be clearly resolved. Then, the sharpening to a 2-m spatial resolution is carried out using the proposed post-processing, resulting in a good retrieval of the hotspot characteristics, as can be observed in Fig. 3(b). The retrieved BGS exhibits a Lorentzian shape with FWHM of $34 \mathrm{MHz}$, as proved in Fig. 3(c) for two typical fiber positions (i.e., inside and 2-m away from the hotspot), in good agreement with the theory.
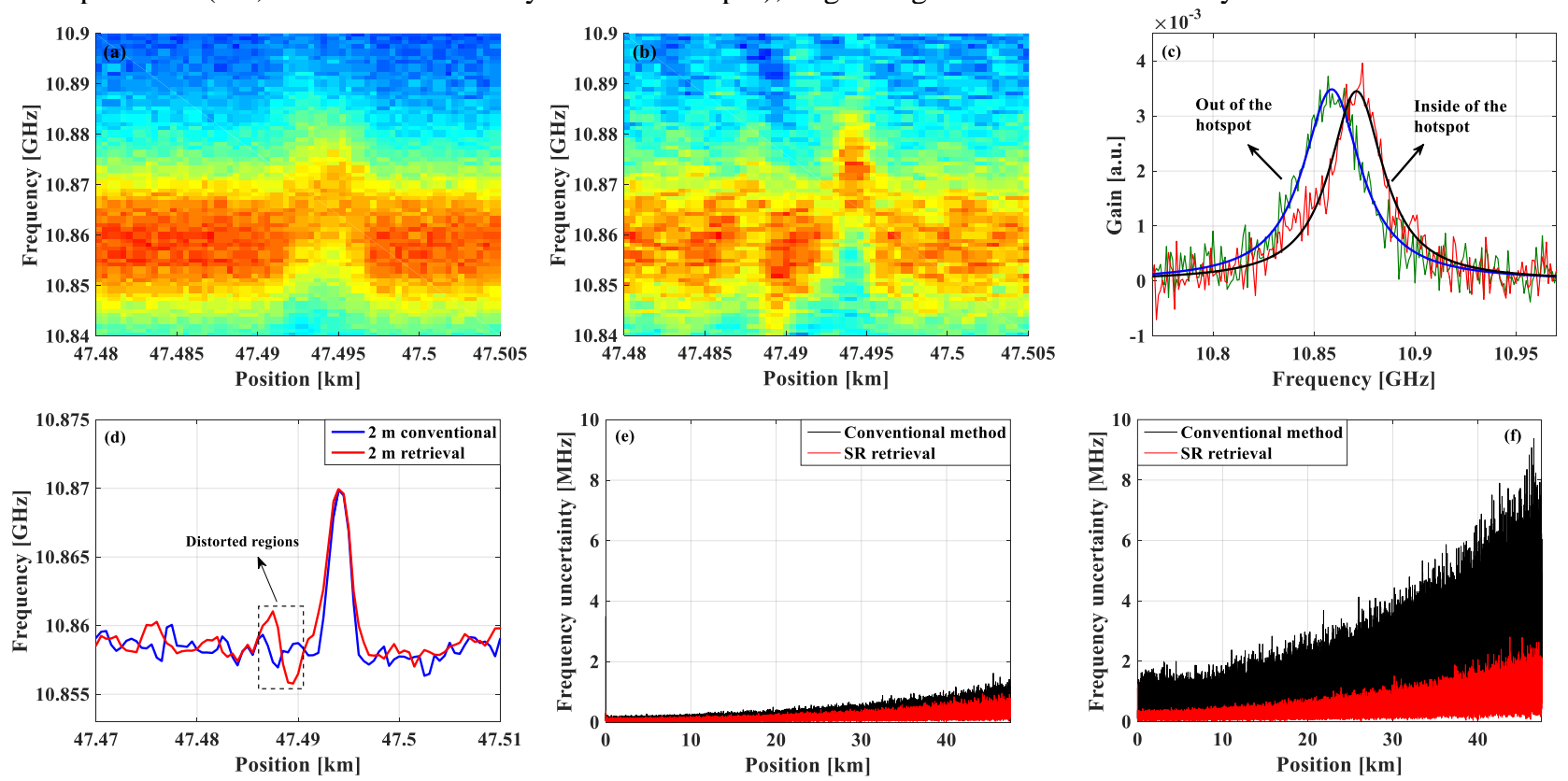

Figure 3. The Brillouin gain distribution as a function of frequency and fiber position with (a): original 6-m SR and (b): retrieved 2-m SR. (c): BGS profiles inside and outside of the hotspot with 2-m SR retrieval; (d) BFS profiles around the hotspot obtained by $2-\mathrm{m}$ pump pulse and by $2-\mathrm{m}$ SR retrieval from a 6-m pump pulse. (e) BFS uncertainty for $2 \mathrm{~m}$ SR. (f) BFS uncertainty for $1 \mathrm{~m} \mathrm{SR}$.

The estimated BFS profile around the hotspot is shown by the blue curve in Fig. 3(b), which matches well with the reference curve (red) taken by a standard BOTDA with 2-m SR, with the notable exception of BFS errors appearing over a short interval prior to the hotspot, as explained in Section 2 of this paper. Since these errors are predictable, they might be reduced making use of the information from the original 6-m SR data together with more complicated processing algorithms. To further validate the performance improvement, the obtained BFS uncertainty along the fiber in the case of 2-m SR retrieval, is shown by the red curve in Fig. 3(e), which outperforms the one using standard BOTDA (black) by a factor 1.56, in good agreement with the theoretical prediction shown in Fig. 2(b). To demonstrate the flexibility of the proposed technique, 1-m SR is retrieved using the same original data at 6-m SR. The resulting BFS uncertainty is compared with results obtained by conventional BOTDA with 1-m SR, as illustrated in Fig. 3(f), showing a 3.5 times improvement that matches the prediction in Fig. 2(b) as well.

In order to validate the capability of a sub-metric SR retrieval, the proposed method is compared with a differential pulsepair (DPP) technique ${ }^{3}$, by carrying out another set of experiments, using a 10.22-km-long sensing fiber with a $20-\mathrm{cm}-l$ long hotspot $\left(55^{\circ} \mathrm{C}\right)$ placed at the fiber end. The long and short pulses for DPP technique are set to $42 \mathrm{~ns}$ and $40 \mathrm{~ns}$, respectively, corresponding to a $20 \mathrm{~cm}$ SR after the differentiation process. The same SR is realized by applying the proposed postprocessing on a single dataset measured using $40 \mathrm{~ns}$ pulse, resulting in a halved measurement time compared to the DPP technique. The retrieved BFS profiles for both techniques are plotted in Fig. 4(a), showing a good agreement except over 
the interval subject to systematic BFS errors. Note that such errors do not show up clearly at other fiber positions although the BFS is non-uniform along the fiber, as shown in the inset of Fig. 4(a). The BFS uncertainty at the fiber end resulting from the proposed technique is illustrated by the red curve in Fig. 4(b), demonstrating a 1.7-fold improvement in BFS accuracy over the DPP technique, associated to a reduced measurement time by a factor 2 .
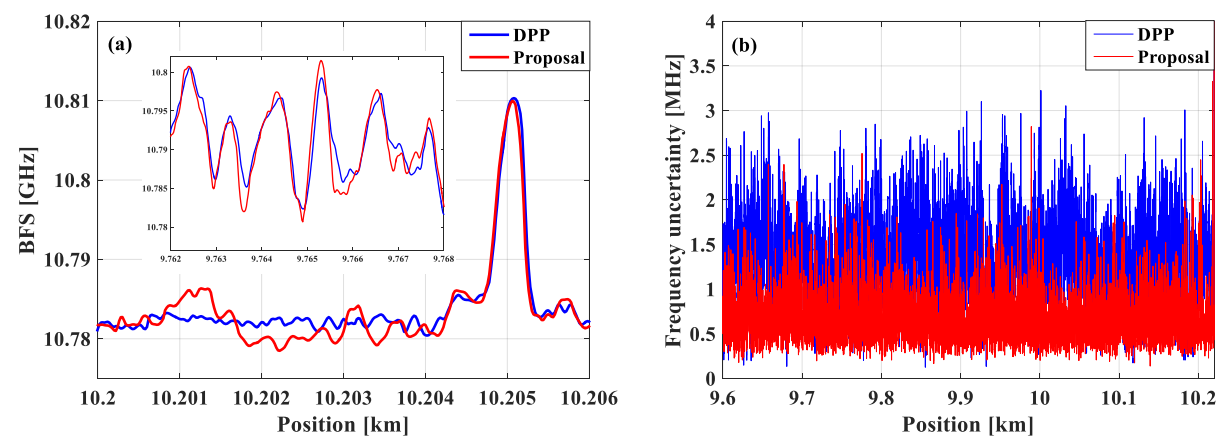

Figure 4. (a) The estimated BFS profiles for the proposal and the DPP method; (b) The BFS uncertainty for the proposal and the DPP method.

\section{CONCLUSION}

A novel post-processing method for retrieving a flexible SR based on a standard BOTDA measurement has been proposed and experimentally demonstrated, offering following advantages:

1) immune to the penalty due to the acoustic inertial response, thus making the response proportional to SR and the BGS FWHM close to natural width;

2) requiring only a standard BOTDA layout with no hardware upgrade, and offering a flexible spatial resolution that can be changed with no new acquisition and adapted during the processing stage according to the response;

3) fast processing since it can be performed in the frequency domain using FFT;

4) no variable and potentially no high speed pulse shaping, but a large enough detection bandwidth compatible with the retrieved SR must be nevertheless maintained;

All the aforementioned advantages come at the expense of systematic BFS errors up to $3 \mathrm{MHz}$ appearing over short fiber sections prior to sharp transitions, which however might be overcome using more sophisticated post-processing algorithms.

Sheng Wang acknowledges the China Scholarship Council (CSC) for supporting his stay at EPFL.

\section{REFERENCES}

[1] Motil, A., Bergman, A. and Tur, M., "State of the art of Brillouin fiber-optic distributed sensing," Optics \& Laser Technology 78, part A, 81-103 (2016).

[2] Alem, M., et al., "Analytical expression and experimental validation of the Brillouin gain spectral broadening at any sensing spatial resolution," 25th International Conference on Optical Fiber Sensors, 103239J (2017).

[3] Li, W., et al., "Differential pulse-width pair BOTDA for high spatial resolution sensing," Opt. Express 16(26), 21616-21625 (2008).

[4] Foaleng, S. M., et al., "High spatial and spectral resolution long-range sensing using Brillouin echoes," Journal of Lightwave Technology 28(20), 2993-3003 (2010).

[5] Beugnot, J. C., et al., "Distributed Brillouin sensing with sub-meter spatial resolution: modeling and processing," Opt. Express 19(8), 7381-7397 (2011).

[6] Zadok, A., et al., "Random-access distributed fiber sensing," Laser \& Photonics Reviews 6(5), L1-L5 (2012).

[7] Sun, X., et al., "Boosting the performance of distributed optical fiber sensors based on adaptive decoder," 26th International Conference on Optical Fiber Sensors, ThE102 (2018).

[8] Soto, M. A., and Thévenaz L., "Modeling and evaluating the performance of Brillouin distributed optical fiber sensors," Opt. Express 21(25), 31347-31366 (2013). 